\title{
NATUKALISM AND ANTI-NATURALISM IN CONTEMPORARY PHILOSOPHY OF SCIENCE
}

\author{
Johan Mouton
}

Department of Philosophy, RAU, Johannesburg

The early sixties saw the rise of more and more schools of thought which came to question the accepted paradigm in the philosophy of science from the 1920's, i.e. logical positivism. What started as a "normal" clash of opinions, eventually developed into, as R.F. Baum called it, the "crisis of the modern intellect". No less than the objectivity or rationality of scientific knowledge became the issue under discussion. On the one hand Kuhn, Feyerabend, et. al. rejected the positivists' conception of rationality as being a reduction of the original meaning of human rationality. According to the "new philosophy of science" rationality has been reduced to logical or methodological computability, thereby neglecting the essential factor of human deliberation and judgement as the essence of human rational behaviour. Logical positivists replied by labelling Kuhn's new emphasis on subjective factors in the scientific endeavour as "irrationalistic" and "relativistic".

It is therefore evident that contemporary clashes of philosophies of science culminated in the fundamental issue of the possibility of reliable human knowledge. The Cartesian ideal of "true and certain" knowledge or the even earlier Greek ideal of "infallible" knowledge, now became the focus of philosophers of science. It can even be said that the broken relationship between philosophy of science and epistemology, has once again been repaired.

The main objective of this article is to analyse the two primary meanings of rationality in the existing debate. It will be shown that the existing differences in meaning can only truly be understood if inte, preted as the outcome of the fundamental difference beıween naturalism (positivisın) and anti-naturalism (historicism). The aforementioned aim is thus broken down to the following:

* First to give a more precise definition of the rather vague concept of "naturalism";

* in the second place to give a short sketch of tnc iwo most important representative paradigms of naturalism and anti-naturalism respectively, i.e. logical positivism and Kuhnian historicism; and

" and in the third place to discuss the meaning of "rationality" as it is understood by these two schools.

\section{WHAT IS NATURALISM?}

In order to clarify some of the meanings of the concept I will introduce a threefold distinction among ontological, epistemological and methodological naturalism.

\section{Ontological naturalism}

According to this thesis the natural world is the only true world - It is Reality. 
The non-natural (God, spirit, mind, idea, etc.) is either seen as apparent realities or reduced to categories in the natural world. The distinctive feature of the thesis of ontological naturalism is its monistic character. According to one of its exponents the naturalist opposes any dualism between Nature and Art or Natural and Supernatural or 'between Natural and Transcendental' ${ }^{1}$. The basic assumption of this thesis is therefore that there is an unbreakable unity in the natural world.

\section{Epistemological naturalism}

This thesis can be stated as follows: The natural world is either the best or the only knowable world. The epistemological naturalist has an unqualified belief that true and certain knowledge of this natural world is within man's reach. The reason for this belief becomes clearer when one analyses their view of the relation bet ween man and nature.

First of all, the natural order is seen as the given, apparent world. Invisible immaterial or spiritual phenomena over and above this given reality do not exist. Secondly, it is assumed that this given reality has its own intrinsic structure which is of necessity good. Any mental construct by man must therefore aim to be as truthful as possible. Pratt writes on this issue that Nature has a character of its own, and "our opinions are true only in so far as they conform to this actual situation"'.

This has the implication that man's role in the whole process of knowing is rather passive. Our mental constructs of reality are at most representations, pictures, impressions or images of reality. Man as knower is not allowed to impress his form on the natural world - Nature puts its stamp on the human mind!

In the final instance it is just this whole passivist attitude that guarantees the truth or certainty of our knowledge. If man abides by the laws of Nature (which are accepted as being unchanging and therefore universally valid), true and certain knowledge is possible. The only cbstacle on the road to Truth, is that man, more often than not, interprets these natural laws wrongly, i.e. our mental constructs are not truthful representations of reality. This is then usually ascribed to some subjective "interference" on the part of man - factors like personal bias, prejudice, values and emotions. Thus: Truth is manifest and attainable by man, but only if man is objective and neutral in his approach to reality ${ }^{3}$.

\section{Methodological naturalism}

In the third instance we find naturalism defending the view that this natural order can only truly be known through the operation of the scientific method. The scientific method comes to be recognized as the only instrument by which truthfu knowledge of reality can be reached. Usually this method is identified with some stringent and formal logical rules of inference, be they inductive or deductive. The important point to make is that the scientific method must be a mechanical procedure - non-subjective and therefore not prone to human fallibility - so as to 
ensure objective knowledge.

\section{Summary}

Naturalism takes its point of departure in the assumption that the natural order constitutes a monistic and unbreakable unity. Because of this assumption (which characterizes all naturalistic philosophies from Bacon to logical positivism), the ideal has always been that this natural unity should be mirrored in any human (mental) reconstruction of this natural order. Because it is further assumed that this order is inherently good, men should at all times conform to this order and not vice versa. If man does approach Nature with an open mind and accepts the given data, Truth is within his reach. It should be kept in mind that man is considered to be part of the natural order. If the causal-mechanistic relations that hold in the main part of Nature can therefore be extended to man's mental processes, the chance of error becomes smaller. Thus: if the process of knowing is seen as the extension of the natural processes to include man's mental processes, the attainment of Truth becomes a much bigger reality. The gap between natural laws and mental constructs must therefore be closed by a mechanical rule/procedure of inference, such as induction or deduction. Already we find here the genesis of the positivists' identification of human rationality with some mechanical means.

\section{LOGICAL POSITIVISM AND KUHN}

One of the interesting points that arises out of a comparison of the work of the naturalism of seventeenth-century Bacon and twentieth-century logical positivism is their very idealistic aims of reforming existing scientific practice. In the same manner that Bacon proposed a Great Restoration of the sciences, the Vienna Circle in their pamphlet in 1929 proposes a reconstruction of existing sciences. The aim of both is to erect the building of science on more reliable foundations

According to the members of the Vienna Circle this task can now (i.e. in the twentieth century) for the first time in the history of science, truly be accomplished, and only as a result of the development of symbolic logic. The task of the philosopher therefore becomes a logical analysis of existing scientific concepts and theories in order to purify science of all metaphysical notions.

The first result of the use of logical analysis, is that a distinction is made between two types of statements:

"To one belong statements as they are made by empirical science; their meaning can be determined by logical analysis or, more precisely, through reduction to the simplest statements about the empirically given. The other statements, to which belong those cited above, reveal themselves as empty of meaning if one takes then in the way that metaphysicians intend"4.

It is, however, not sufficient to show that statements that are made by empirical science are the only meaningful statements. The ideal of science through the years 
has been the attainment of true and certain statements. The latter part of their manifesto therefore is directed to the objective of justifying their belief in the truth and certainty of statements made by the empirical sciences. In true Humean tradition a distinction is made between two layers of knowledge in empirical science. (What Feigl has called the two-tier view of science.) 5 . First of all there is the basis or foundation which contains the so-called Protocol sentences which refer directly to the empirical data. In accordance with the thesis of epistemological naturalis $m$ it is accepted that these Protocol sentences are acceptable because they are only the symbolic representation of what has been given or received in observation. "That knowledge of the world is possible rests not on human reason impressing its form on the material, but on the material being ordered in a certain way."6

Thus. The credibility of the foundational sentences is guaranteed through its empirical reference. Upon this foundation a superstructure of theoretical sentences is erected. Again, in the spirit of methodological naturalism, the credibility of these high-level theoretical sentences is guaranteed through their reduction to the - foundational sentences. "Since the meaning of every statement of science must be statable by reduction to statement about the given, likewise the meaning of any concept, whatever branch of science it may belong to, must be statable by stepwise reduction to other concepts, down to the concepts of the lowest level which refer directly to the given"?.

It is interesting to note that the further developments after 1929 more or less focussed on these two aspects of the naturalistic, and therefore the positivist, position. On the one hand there was the problem of the relation between the basic/foundational or Protocol sentences and the empirical given.

On the other hand there was the problem of the gap between Protocol sentences and the high-level theoretical superstructure. Already in the early thirties there was a long debate between Carnap and Neurath concerning the first issue, i.e. whether the Protocol sentences should be understood in a phenomenalistic or physicalist sense. The other problem was first given serious attention in 1936 when Carnap, in his "Testability and Meaning" changed the criterion of verification to a confirmation-criterion. Since then there have been two main developments concerning this very problem; the one was Carnap's programme of an inductive logic; the other the attempt by the Bayesians like Salmon, Hesse and Maxwell, to interpret confirmation not so much as a logical function, but much more as a function of personal belief or credence. It can be safely stated today that both these attempts have proved to be futile.

I now proceed to discuss Kuhn's philosophical position very briefly.

Since Kuhn appeared on the scene in the early sixties and brought about a revolution in the philosophy of science in his own way, it has been a favourite topic in philosophy of science journals to try and find the real common factor in the so-called "new philosophy of science", i.e. "What is the common denominator in 
the works of people like Hanson, Toulmin, Feyerabend, Polanyi, Kuhn and even people like the later Wittgenstein?".

The thesis that I would like to defend (as stated in the introductory paragraphs), is that their common critique of different aspects of logical positivism can be generalised as a new torm of anti-naturalism. First of all I will trv to prove my point through a short outline of Kuhn's position, and in the second place I will try to show in the third part of the article, that this difference can best be seen in the different meanings that the naturalists and anti-naturalists attach to the concept of "rationality"

Kuhn approaches the philosophy of science primarily as a historian of science. This very fact can't be emphasized too much. Whereas the positivists ignored the genesis and dynamics of scientific theories because of the fact that the so-called context of discovery is really the domain of psychology, Kuhn was primarily interested in the dynamic growth of scientific theories. In his study of the process he came to the conclusion that the positivistic explanation of the history of science as growththrough-accumulation was false and that the history of science must rather be understood as the succession of periods of normal and revolutionary scientific practices.

The characteristic feature of the normal science phases is that science is practised in a particular paradigm or Weltanschauung. A paradigm, according to Kuhn, can be defined as a set of theories and values to which a certain scientific community is committed. The acceptance of such a common research tradition, entails the acceptance of some common values and criteria which are used in normal scientific practice. The specific paradigm in which you work also determines how you see the worid, how you select your facts and data, as well as the specific meaning you assign to different concepts.

The appearance of some anomalies, i.e. theoretical and observational discrepancies which can't be explained by the existing paradigm, usually heralds the rise of a new paradigm. Because of the all-pervasive influence of a paradigm, it is understandable that the replacement of one paradigm by a new one, is sketched as a scientific revolution. This leads Kuhn to make the conclusion that two successive paradigms are essentially incompatible.

Because Kuhn's philosophy is very well-known today, this short exposition will suffice ${ }^{8}$. It remains for me, however, to show in what ways Kuhn can be described as an anti-naturalist.

The first thesis of naturalism, i.e. ontological naturalism, implies that there is only one, unchanging, uniform, natural world. Although I could not find any reference in the works by Kuhn to the ontological status of the natural world, it is, however, possible to infer the following from his position. Because every paradigm determines my perception of the natural order, every new paradigm does imply a new and different "perceived reality". This philosophical position probably comes 
nearest to that of another earlier anti-naturalist, i.e. Immanuel Kant with his distinction between a phenomenal and noumenal world.

This has the second implication that Kuhn inadvertently also rejects the thesis of epistemological naturalism. Because Kuhn lends priority to the paradigm in the relation between paradigm and nature in the sense that the paradigm determines one's perception of the world, one's selection of data, the criteria one accepts in problem-solving, etc., one could say that the paradigm constitutes a new conceptual world.

In The Structure of Scientific Revolutions Kuhn writes the following: “ Mopping-up operations are what engage most scientists throughout their careers. Thev constitute what I am here calling normal science. Closely examined, whether historically or in the contemporary laboratory, that enterprise seems an attempt to force nature into the performed and relatively inflexible box that the paradigm supplies" Concerning the first aspect of the thesis of epistemological naturalism Kuhn therefore rejects the view that concepts and theories are only representations of an - unchanging natural world. This view of the character of theories will in the second place also have implications for the problem of truth. In a response to Popper Kuhn states the following: "Sir Karl takes it for granted that the proponents of competing theories do share a neutral language adequate to the comparison of such observation reports. I am about to argue that they do nct. If I am right, then 'truth' may. like "proof' be a term with only intra-theoretic applications"10. Kuhn then goes on to discuss the whole problem of the incommensurability of successive paradigms, and concludes as follows: "In the absence of a nutral language, the choice of a new theory is a decision to adopt a different native language, and to deploy it in a correspondingly different world. That sort of transition is, however, not one which the terms 'choice' and 'decision' quite fit"'.

To summarise: Kuhn is compelled, in a sense, because of his particular view of paradigms and their relation to nature, to reject the view that truth is something universal, objective and therefore inter-paradigmatic. At the most Kuhn is prepared to defend the intra-paradigmatic goal of truth and especially then as the continued solving of problems.

The most important of Kuhn's critiques of positivism can, however, be found in his rejection of the thesis of methodological naturalism. As you will remember this thesis amounts to the belief that the scientific method is the only instrument through which true and certain knowledge of reality can be searched. The other important point that we made was that the scientific method is usually seen as a mechanical procedure whereby scientific and non-scientific knowledge can be demarcated. Already on the third page of TSOSR, Kuhn remarks that one of the results of his historical study is to show, and I quote, "The insufficiency of methodological directives, by themselves, to dictate a unique substantive conclusion to many sorts of scientific questions"12. In another article he writes that "There can be no set of rules of choice adequate to dictate desired individual behaviour in the concrete cases that scientists will meet in the course of their careers"13 
Kuhn therefore explicitly rejects the positivist and also naturalist ideal of a strictly mechanical (or logical) procedure which will decide as it where on behalf of the scientist between scientific and non-scientific knowledge. According to Kuhn it is the scientist's commitment to certain paradigmatic values that helps him to choose acceptable scientific theories.

In conclusion, Kuhn's position is thus: Because of the inverted relation between paradigm and changing reality, as well as the inner complexity of the paradigm's structure, it is unrealistic to believe (like the positivists do) that an analysis of the formal relation between theory and evidence can give us an explanation of why one theory is replaced by a new one. It is only when one takes into consideration inter-paradigmatic relations and especially the paradigm-induced values that scientists accept in times of theory-choice that one can get a better understanding of scientific evaluation.

\section{RATIONALITY}

This brings me to my last and final point, i.e. the meaning of rationality in the positivist and Kuhnian paradigms.

Since the earliest philosophizing about problems of knowledge, the search for knowledge has been synonymous with the search for infallible knowledge. The central role in philosophy of the quest for infallibility is equally well illustrated by the persistent search for some unequivocal foundation on which the edifice of knowledge can be built. We have examined the logical positivists' attempt to take the empirically given as the foundation of knowledge. It is, however, pertinent to take a closer look at the other pillar of knowledge in the positivists' programme, i.e. the inference from the superstructure to foundational sentences. We have seen earlier that an important implication of the epistemological naturalist's point of view is the elimination of all human, and by definitioin, subjective factors from the scientific process. This aspect finds it most evident effect in the positivists's distinction between the contexts of discovery and justification. By limiting the legitimate domain of the philosophy of science to the latter, the positivist was just following the tradition of replacing fallible human judgement by a set of algorithms. I quote Harold Brown to the effect that "This ideal controlled early logical positivist ideas on the verification of theories, receiving its most extreme expression in Wittgenstein's attempt to reduce all propositions to truth fuctions of atomic propositions. ... This programme has, we have seen, been abandoned and replaced, among logical empiricists, by the search for an inductive logic based on probability theory Again the project is to find an alogorithm on the basis of which we can evaluate scientific theories, the assumption being that even if we cannot prove the final truth of an hypothesis, we can produce a set of rules which will allow us to determine the degree to which it has been confirmed by the available evidence"14.

Brown then makes the very relevant remark that "The attempt by logical 
empiricists to identify rationality with algorithmic computability is somewhat strange, since it deems rational only those human acts which could in principle be carried out without the presence of a human being!"15. If one takes Brown's argument to its logical conclusion it means that to be rational in the positivist's meaning of the word, is to act non-rationally (i.e. without using your mind). Brown, however, takes another line: According to him it is even, with all the algorithms in the world, possible to act irrationally, in the positivists' sense of the word. "There are many different directions in which the scientist can proceed in attempting to deduce testable consequences from his hypothesis, each of which may be strictly in accordance with a set of algorithms, but he has no algorithm for determing which line to pursue. An informed judgement is required and it is in making such judgements that we must rely on reason. As long as decisions can be carried out by means of algorithms, human intervention is not necessary, it is exactly when we have no effective procedures to guide us that we must turn to an informed, rational human judgment" ${ }^{\prime \prime}$.

If we turn to Kuhn now, we see that it is precisely because of his historical survey of scientific theories that he came to reject the positivist concept of rationality. Both he and people like Feyerabend and Holton have conclusively shown, I think, that there is no clear, simple relation between the results $\mathrm{cf}$ experiment or observation and scientific theories. Even in the simplest, most straightforward instance, i.e. the case of an observational result which contradicts a theory, the practising scientist is not hound automatically to reject part of his theory. The decision as to how a discrepancy between theory and observation is to be handled requires a judgement by scientists. This, I take it, is the thrust of some of Kuhn's most widely attacked claims, e.g. that such questions (of theory choice) "can never be settled by logic and experiment alone" and "the competition between paradigms is not the sort of battle that can be resolved by proofs".

To understand Kuhn's concept of rationality better, Brown suggests that one should compare Kuhn's position with Aristotle's man of practical wisdom as he is sketched in his Ethics. For Aristotle, ethics is concerned with human behaviour and because of the complexity of human behaviour, there are no first principles on the basis of which to construct a science. Ethical decisions require deliberation, the ability to weigh information and make decisions in cases in which there is no necessary knowledge. The conclusion is not infallible and there is no guarantee that all adequately informed people who deliberate on an issue will reach the same decision, but this does not make the decision arbitrary or irrational. While Aristotle's man of practical wisdom, i.e. the man who has had sufficient experience to understand human behaviour and has developed his ability to deliberate, offers a model of individual rationality, Kuhn uses, I think, basically the same model of rationality - only on the level of the community of scientists. In a well-known statement Kuhn gives a description of the process of communal deliberation: "Take a group of the ablest available people with the most appropriate motivation; train them in some science and in the specialities relevant to the choice at hand; imbue them with the value system, the ideology, current in their discipline and, finally, let them make the choice"17. 
Brown concludes that "It is the consensus of the workers in a discipline that determines what constitutes knowledge in this disciplıne, but the group may later discover that it made a mistake. The group is no more infallible than the individual (but this does not mean that it is as fallible as the individual"'s.

It should be evident by now in what way Kuhn's concept of rationality is also the result of his particular anti-naturalistic point of view.

Because Kuhn rejects the main thesis of naturalism, and proceeds on the basis of the priority of some theoretical structure, i.e. a paradigm, it could be interpreted in a negative way, as a restriction of scientist's activities. Scientists' whole perceptual, conceptual and even semantic make-up is already laid down by the paradigm to which they are committed. If one looks at it, however, from the point of view of decision-making, the adherence to a paradigm seems to become more and more attractive. Because a paradigm dictates certain ways of doing things, because a paradigm provides common criteria and values that can be utilised in times of crisis, the possibility of consensus is so much greater. The reason why group decisions throughout the history of science, seem to be relatively unanimous can then be understood as a function of the paradigms that scientists adhered to.

In conclusion I think that the main lesson to be learned, can be found in the tradition of Western Philosophy to identify knowledge with infallibility and truth. If one accepts knowledge to be reasonable or even justified belief, it does not entail the further thesis, t.e. that knowledge should necessarily be true and infallible. What counts as a reasonable belief will certainly differ from time to time, and from context to context If this is seen as relativism, then all human knowledge will be relativistic.

\section{REFERENCES}

1. Randall. J.H. 1944. The nature of naturalism, p. 357.

2. Pratt, J.P. 1939. Naturalism, Greenwood Press, Connecticutt, p. 3-4.

3. The thesis of epistemological naturalism is in many aspects similar to Popper's "Theory of Manifest Truth". See his Conjectures and Refutations, 1974, Sth ed., London, Routledge and Kegan Paul, p. 5.

4. 1929. Wissenschaftliche Weltauffassung: Der Wiener Kreis, D. Reidel Publ. Co., Dondrecht, Holland, p. 8-9.

5. The 'orthodox' view of theories: Remarks in defense as well as Critique. (In Radner \& Winokur, Analyses of theories and methods of physics and psychology; University of minnesota Press), p. 3-16.

6. Wissenschaftliche Weltauffassung, p. 14-15.

7. Idem, p. 11.

8. For further articles on Kuhn in South African journals, see my articles in Perspektief. 16(1):38-53, 1977. (Paradigma: Ideologie of nie?) and N.T. van der Merwe, Paradigm, science and society, Koers, $40(4,5 \& 6): 328-358), 1975$.

9. 1970. The Structure of Scientific Revolutions. The University of Chicago Press, Chicago p. 24. 
10. Lakentos \& Musgrave. 1970. Reflections on my critics. Criticism and the growth of knowledge, Cambridge University Press, Cambridge, p. 265-266.

11. Idem, p. 277.

12. The Structure of Scientific Revolutions, p. 3.

13. Reflections on my critics, p. 238.

14. 197T. Perception, theory and commitment, Precedent Publ. Inc., Chicago, p. 146.

15. Idem, p. 147-148.

16. Idem, p. 148.

17. Reflections on my critics, p. 237-238.

18. Perception, theory and commitment, p. 150. 